\title{
Los logotipos de privacidad en Internet: percepción del usuario en España
}

\author{
David López Jiménez ${ }^{1}$, Andrés Redchuk ${ }^{1}$, Eduardo Carlos Dittmar ${ }^{1}$, Jenny Patricia \\ $\operatorname{Vargas}^{1}$ \\ david.lopez@uautonoma, andres.redchuk@autonoma.cl,eduardo.dittmar@uautonoma.cl, \\ jenny.vargas@uautonoma.cl \\ ${ }^{1}$ Universidad Autónoma de Chile, Av. Pedro de Valdivia 641, CP. 7500138, Santiago de Chile, Chile.
}

DOI: 10.4304/risti.12.49-63

\begin{abstract}
Resumen: En este trabajo analizamos la relevancia que la privacidad tiene en la era digital. Representa uno de los aspectos susceptibles de generar desconfianza en el consumidor o usuario que recurre a Internet. Para garantizar que las empresas que operan en la Red son respetuosas con los derechos de los usuarios, se han ideado logotipos acreditativos de la adhesión a un elenco de buenas prácticas en el ámbito de la privacidad. Uno de los objetivos fundamentales del presente artículo es poner de relieve que tales instrumentos constituyen un útil complemento de la normativa legal. Igualmente se realiza un estudio empírico, sobre la base de una entrevista semiestructurada abierta, dirigido a conocer los efectos que estos iconos tienen respecto a sus destinatarios. Hemos delimitado el presente estudio a los logotipos que operan en España, con independencia del origen de los mismos, dado que algunos son foráneos.
\end{abstract}

Palabras-clave: confianza; confidencialidad; Internet; privacidad; sellos.

\section{Internet privacy seals: user perception in Spain}

Abstract: In this paper we analyze the importance that privacy has in the digital age. It represents one of the aspects which can generate distrust in the consumer or user of Internet. In order to guarantee that those companies which operate in Internet are respectful of the user's rights, it has been designed logos for the adherence to a list of good practices in the field of privacy. One of the most important objectives of this paper is to emphasize that such instruments provide a useful complement to the legal regulations. However, it will be done an empirical study, based on the open semi-structured interview, and designed to determine the effects that these icons have on their receivers. We have delimited the present study to logos operating in Spain, regardless of their origins, because some of them are outsiders.

\footnotetext{
${ }^{1}$ Este trabajo forma parte del Proyecto de investigación FONDECYT No 11130188.
} 
Keywords: trust; confidentiality; Internet; privacy; seals.

\section{Introducción}

El recurso a Internet para realizar transacciones, de diversa índole, representa una modificación significativa de la forma tradicional de efectuar negocios (Teece, 2010). Podemos, en cierta medida, indicar que, en la actualidad, constituye, dada su relevancia, más que una opción, una obligación para las empresas. Para hacer frente a las situaciones que suscitan desconfianza en la Red, se han creado los logotipos de calidad -que bien pueden aludir, de manera exclusiva, a la privacidad o bien referirse de manera complementaria a la misma- que se incorporan a los sitios Web de las empresas comprometidas con los mismos. Tal decisión, de carácter íntegramente facultativa, tiene como objetivo elevar la confianza de quienes los visionen que son los potenciales consumidores o usuarios (Cashell, 1999). Asimismo, debe considerarse que generalmente los mismos suelen fundamentarse en un código de conducta escrito.

Con estos antecedentes, deben precisarse los que serán los grandes puntos del presente artículo. En primer término, se efectuarán ciertas apreciaciones sobre la privacidad en la era digital. Posteriormente, se verá el concepto, caracteres y diversas modalidades de sellos de privacidad en el ámbito digital. Para tomar conciencia de sus efectos, se ha optado por efectuar un estudio empírico en el que, tomando como base una entrevista semiestructurada abierta, se analizarán los efectos que la adhesión de este tipo de logotipos gráficos comporta para los principales destinatarios de los mismos que son los consumidores o usuarios. Debe reseñarse que hemos consultado a los usuarios sobre una serie de aspectos, que se detallarán en el estudio empírico, respecto a los sellos más significativos que operan en el escenario español. Estos últimos no son únicamente de origen español (Confianza Online, Agace, Optima Web, Aenor, E-Confia y Euro-label España), dado que ciertos prestadores de servicios radicados en España han asumido el compromiso de exhibir logotipos de privacidad que tienen su sede en otros países como los Estados Unidos (Truste, BBB Online y Web Trust). El papel que los mismos juegan, como se verá, resulta determinante, siendo sensiblemente valorada su presencia en un sitio Web.

\section{La privacidad en el espacio digital}

Las nuevas tecnologías tienen incidencia sobre numerosos derechos en el que ocupa una posición destacada la privacidad (Caudill \& Murphy, 2000; Zhan \& Rajamani, 2008). Los datos de carácter personal, en la actualidad, tienen un extraordinario valor. Los perfiles formados tienen un precio de compra/venta muy atractivo en el mercado, y lo que es peor, se trata de una actividad que es completamente invasiva a nuestra intimidad, ya que muchas veces se trata de una acción que no ha sido ni conocida ni consentida (Jawahitha, 2004).

Existe un importante número de investigaciones que estudian la repercusión que la privacidad tiene sobre la adopción del comercio electrónico $\mathrm{B} 2 \mathrm{C}$, como alternativa posible en la compra de bienes o servicios (Sirkka et al, 2010; Aljifri, Pons \& Collins, 2003). Se ha llegado a la determinación de que la preocupación o intranquilidad, en materia de privacidad electrónica, es uno de los elementos que más repercute en la 
falta de confianza del consumidor o usuario del comercio electrónico (Belanger, Hiller \& Smith, 2002).

La privacidad está unida a las medidas que, en materia de instrumentos de seguridad electrónica, decidan instaurarse para la protección, integral y continuada, de los datos de carácter personal de los potenciales consumidores o usuarios (Raab, 1997). El papel que el Derecho ha de desarrollar, en toda esta cuestión, es ciertamente relevante. Sin embargo, en ciertas ocasiones, surgen lagunas legales cuya repercusión puede resultar significativa no sólo para los implicados, sino, lo que es más importante, para la sociedad en general. En estas circunstancias, se erigen, como solución paradigmática, los códigos de conducta -cuyo compromiso se pone de relieve mediante la exhibición de un logotipo gráfico- que garantizan, además del cumplimiento que, en materia de privacidad, se exige, por la legislación aplicable, un plus adicional de amparo en claro beneficio del consumidor o usuario. En todo caso, ha de tomarse conciencia que no solo contribuyen a tal confianza estos últimos logotipos, ya que debe considerarse, por ejemplo, la redacción de políticas de privacidad y políticas de uso por parte de los prestadores de servicios. Aunque pueden incorporarse de manera conjunta en el sitio Web, también pueden hacerse constar de forma individual.

\section{Los logotipos gráficos en el ámbito de la privacidad}

En el presente apartado efectuaremos un examen de los diversos logotipos gráficos que se han ideado para tutelar la privacidad de los usuarios. A este respecto, en primer término, acometeremos su delimitación conceptual, las diversas modalidades que de los mismos existen, así como el procedimiento establecido para realizar la adhesión a este tipo de sugerentes figuras. Finalizaremos, efectuando un breve análisis comparativo de las buenas prácticas relativas a la privacidad que efectúan los diferentes documentos en los que se fundamentan los sellos gráficos. Nótese, en este sentido, que nos centraremos, de manera exclusiva, en los logotipos que operan en España, con independencia del origen de los mismos, dado que algunos, como se verá, son foráneos.

\subsection{Delimitación conceptual}

Para facilitar la distinción positiva, respecto a las empresas comprometidas con actuaciones empresariales de excelencia que, además de observar la legalidad, reconocen un plus tuitivo adicional -diferenciándose de la competencia- para los potenciales consumidores o usuarios establecidos en documentos de buenas prácticas -generalmente comprendidos en códigos de conducta-, la industria, ha ideado mecanismos de acreditación de esa vinculación. Nos referimos a la posibilidad de exhibir iconos gráficos, logotipos o, en suma, sellos de calidad.

Son numerosas, y muy diversas entre sí, las definiciones que se han operado en relación a la cuestión que nos ocupa. En efecto, un sector de la literatura académica (McKnight, Cummings \& Chervany, 1998) considera que representan iconos que ponen de relieve que un empresario se compromete a respetar las mejores prácticas comerciales sobre una determinada materia. Otros, en un sentido similar, entienden que representan signos perceptibles para los compradores que manifiestan que un determinado comerciante que opera en el plano digital se ha comprometido con ciertas 
reglas de fiabilidad dadas por una organización o institución que, de alguna forma, fomenta el icono gráfico (Kimery \& McCord, 2006).

Tales logotipos son concebidos y, ulteriormente, expuestos con el objetivo de generar ciertas consecuencias en las actitudes del destinatario. La labor que tal icono cumplirá es, además de distintiva frente a los competidores, de calidad (Trites, Boritz \& Pugsley, 2006), pues su utilización, frente a los potenciales consumidores o usuarios, pone de relieve que el empresario respeta los estándares de calidad -normativos y éticosestablecido en el código de conducta al que se ha adherido el prestador de servicios.

Los sistemas que fomentan la confianza en el plano digital son eficaces para aumentar las transacciones comerciales (Ho \& Oh, 2009). Así se ha manifestado (Kaihong \& Mingxia, 2007), que el empleo de los logotipos de calidad es una de las estrategia más plausibles, a efectos empresariales, que pueden coadyuvar al crecimiento de las pequeñas empresas y compañías emergentes en la Red (Noteberg, Christiaanse \& Wallage, 2003). Repárese en que estas últimas, a diferencia de las que están consolidadas, son desconocidas por los potenciales usuarios.

En el supuesto de que los logotipos gráficos relativos a la privacidad y otros aspectos concomitantes sean conocidos, por parte de sus destinatarios, la presencia en el sitio Web puede determinar que la transacción se opere en un sentido favorable para el comerciante que los exhiba (Odom, Kumar \& Saunders, 2002). Ahora bien, no todos los estudios que se han operado sobre los logotipos de calidad, que resulta extensivo a la privacidad, se posicionan a favor de su eficacia positiva (Kim, Ferrin \& Rao, 2008). En todo caso, debemos valorar, en sus justos términos, las consecuencias desfavorables que de tales investigaciones se derivan. En ocasiones, los resultados negativos pueden obedecer al hecho de que se realizan en un contexto ficticio. Nos referimos, a título de ejemplo, al hecho de que la muestra de estudio se refiera a estudiantes universitarios que pueden estar poco familiarizados con los instrumentos que analizamos (Mcknight, Choudhary \& Kacmar, 2004). En cualquier caso, existen autores que critican esta última afirmación, estimando, sin embargo, que los alumnos de centros universitarios constituyen una muestra ciertamente útil, ya que, entre otros factores, suelen tener cierto grado cultural y, por ende, un nivel superior a la media (Lala et al., 2002).

\subsection{Modalidades}

Existe una multiplicidad de logotipos de calidad en el espacio digital. Los más numerosos se han ideado en materia de privacidad y seguridad -que, aunque estén relacionados, pueden concebirse de manera independiente-. Lo que se busca en definitiva es garantizar a todos los usuarios que las transacciones efectuadas en el sitio Web que los exhiben pueden entenderse seguras y, en su caso, privadas. Ambas tipologías de logotipos -privacidad y seguridad- cumplen dos importantes funciones. Por un lado, ponen de manifiesto un grupo de buenas prácticas sometidas a normas aceptables o estándares en el caso de la privacidad. Y, por otro lado, están relacionados con las percepciones de riesgo asociadas a un sitio Web, favoreciendo, de esta manera, la divulgación de información efectuada por los usuarios (Miyazaki \& Krishnamurthy, 2002). En este último sentido, debe repararse en que si los consumidores se sienten seguros (en lo que debe estimarse incluido las buenas prácticas relativas a la privacidad 
del usuario) en un determinado sitio Web confiarán, en mayor medida, en el mismo (Gefen, Karahanna \& Straub 2003).

Esta modalidad de logotipos, relativos, con carácter específico, a la privacidad y a la seguridad, coexiste con otros que acreditan la observancia de elevados niveles de excelencia más allá de tales extremos. La tipología de iconos a los que apuntamos, garantizan, entre otros aspectos, la tutela de los menores de edad, el recurso a técnicas publicitarias no invasivas, normas de accesibilidad, la operativa de las transacciones virtuales y la resolución extrajudicial de conflictos en línea.

Hemos optado por elaborar un cuadro -tabla 1- en la que podrá visualizarse la diversidad de sistemas de autorregulación que han sido ideados en el espacio digital, denominaciones, direcciones electrónicas, así como si cuentan o no con organismo de control o sistema de resolución extrajudicial de conflictos (Alternative Dispute Resolution -ADR-). Se han incluido los más representativos de Europa -y, por ende, España-. En el supuesto de que el sistema de autodisciplina no presente órgano de control -encargado de verificar su observancia-, la vigencia de las previsiones relativas a la privacidad en el código de conducta -en el que se fundamenta el sello de calidad-, por parte de los adheridos, parece más bien testimonial. En otras palabras, frente al incumplimiento del articulado incluido en el mismo, no cabrán sanciones, por lo que su fuerza vinculante es inapreciable. En esos casos, podríamos afirmar que, más que una herramienta de verificación real del respeto de la privacidad en Internet, representa simplemente un instrumento de marketing.

Tabla 1 - Enumeración de los sellos de confianza por país

\begin{tabular}{|c|c|c|c|}
\hline País & Denominación & URL & ADR \\
\hline \multirow[t]{6}{*}{ Alemania } & Bonicert & $\underline{\text { www.bonicert.de }}$ & Sí \\
\hline & EHI Euro-Label y EHI bvh Label & www.shopinfo.net & Sí \\
\hline & Internet Privacy Standards & www.datenschutz-nord.de & No \\
\hline & Safer Shopping & www.safer-shopping.de & Sí \\
\hline & Shoplupe & $\underline{\text { www.shoplupe.com }}$ & No \\
\hline & Trusted Shops & www.trustedshops.de & Sí \\
\hline Austria & Gütezeichen & www.guetezeichen.at & Sí \\
\hline \multirow[t]{2}{*}{ Bélgica } & Becommerce label & www.becommerce.be & Sí \\
\hline & Unizo & www.unizo.be & No \\
\hline Dinamarca & E-Market & www.e-maerket.dk & Sí \\
\hline \multirow[t]{6}{*}{ España } & Aenor & www.aenor.es & Sí \\
\hline & Agace & www.agace.com & Sí \\
\hline & Confianza Online & www.confianzaonline.es & Sí \\
\hline & E-Confia & $\underline{\text { www.econfia.com }}$ & Sí \\
\hline & Euro-Label España & www.euro-label.com/es & Sí \\
\hline & Optima Web & www.optimaweb.anetcom.es & Sí \\
\hline
\end{tabular}




\begin{tabular}{|c|c|c|c|}
\hline País & Denominación & URL & ADR \\
\hline \multirow[t]{2}{*}{ Francia } & Fia-Net & www.fia-net.com & Sí \\
\hline & Labelsite & www.labelsite.org & Sí \\
\hline Grecia & Epam & www.enepam.gr & No \\
\hline Holanda & Thuiswinkel & www.thuiswinkelwaarborg.nl & Sí \\
\hline Hungría & eQ-recommendation & www.ivsz.hu & No \\
\hline \multirow[t]{2}{*}{ Irlanda } & EIQA W-Mark & www.eiqa.com & No \\
\hline & Segala Trustmark & www.segala.com & No \\
\hline \multirow[t]{5}{*}{ Italia } & E-com-quality-mark & www.e-com-quality-mark.it & No \\
\hline & Euro-Label Italy & www.euro-label.org & Sí \\
\hline & Fiducia & www.progettofiducia.it & Sí \\
\hline & NetQuality & $\underline{\text { www.isec.it }}$ & No \\
\hline & Q-Web & www.qwebmark.it & No \\
\hline Luxemburgo & E-commerce certified & www.e-certification.lu & Sí \\
\hline \multirow[t]{2}{*}{ República Checa } & Apek & www.apek.cz & No \\
\hline & Soap & www.spotrebitele.info/audit & No \\
\hline Malta & Euro-Label Malta & $\underline{\text { www.eurolabel.gov.mt }}$ & Sí \\
\hline \multirow[t]{2}{*}{ Polonia } & E-Commerce ILiM Certyfikat & www.ilim.poznan.pl/index eng.php & Sí \\
\hline & Trusted Store & www.sklepy24.pl & No \\
\hline Portugal & PACE & www.comercioelectronico.pt & Sí \\
\hline \multirow[t]{4}{*}{ Reino Unido } & SafeBuy & www.safebuy.org.uk & Sí \\
\hline & TrustMark & www.trustmark.org.uk & Sí \\
\hline & TrustUK & www.trustuk.org.uk & No \\
\hline & WebtraderUK & www.webtraderuk.org.uk & Sí \\
\hline
\end{tabular}

Como puede inferirse, hay una notable multiplicidad en los sistemas de autodisciplina. Lo más recomendable, en cualquier caso, sería realizar un esfuerzo en beneficio de la racionalización del sistema. Un sistema de autodisciplina relativo a la privacidad podrá ser realmente eficaz cuando sea ampliamente conocido en la sociedad a la que se dirige. La existencia de un número más reducido de iniciativas sobre la privacidad ayudaría a que éstas ostentaran un mayor conocimiento por parte del público. Por lo contrario, un gran número de las iniciativas darían como consecuencia un horizonte opuesto, es decir, produciría en los usuarios una profundización en el desconocimiento general de este tipo de normativas.

\section{3•3. Procedimiento de adhesión}

Estas normas, que son de carácter voluntario, son confeccionadas por una determinada entidad promotora, estando la obediencia del código de buenas prácticas a cargo de un 
organismo de control. Al ser de carácter opcional para el Prestador de Servicios de la Sociedad de la Información -PSSI- que actúa en Internet, éste debe ser quien requiera, de manera expresa, su adhesión. El procedimiento, que consta en el gráfico 1, requiere la solicitud de adhesión expresa del empresario. A continuación, se analiza la documentación aportada por el PSSI, procediéndose a valorar la conformidad del sitio Web a las estipulaciones establecidas en el código ético. En el articulado de este último se incluirá, bien de manera íntegra bien en algunas de sus reglas, el deber de observar un elenco de buenas prácticas relativas a la privacidad. De tal verificación se pueden derivar dos alternativas. La primera que el sitio Web en cuestión sea plenamente garantista con el código de conducta -en cuyo articulado se incluyen previsiones en materia de privacidad- y la segunda que no sea así, y que, por consiguiente, haya que realizar algún cambio. Tras la actuación en este último sentido, la entidad promotora, nuevamente, valorará si, en su caso, procede la autorización para mostrar el sello de confianza acreditativo del compromiso a un determinado código de buenas prácticas. Este último deberá constar, ya que es su razón de ser, en los diversos espacios que formen parte del sitio Web. Es decir, resulta conveniente que, para desplegar toda su eficacia, figure, no sólo en la página de inicio -homepage-, sino en todos los apartados susceptibles de ser visualizados por el potencial consumidor o usuario. De otra manera, no se garantizaría que este último (verdadero destinatario) visualizase la imagen integrante del sello de confianza y lo que, por tanto, ello supone en el campo concreto de la privacidad.

Gráfico 1 - Proceso de adhesión a un determinado logotipo de privacidad

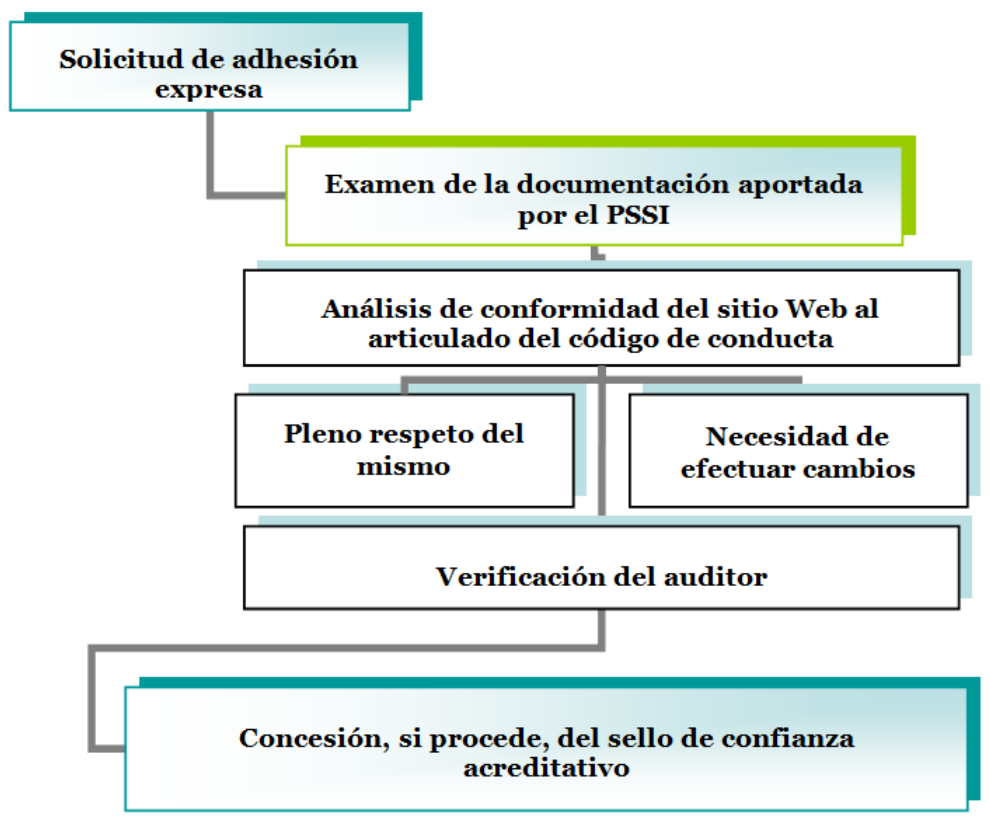




\subsection{Heterogeneidad respecto a la ordenación de la materia}

Los iconos de calidad regulan, en distinto grado, diferentes extremos relativos a la privacidad. Esta última junto con la seguridad son dos de los elementos que más preocupan a los potenciales consumidores o usuarios en Internet. Conscientes de la importancia de ambas, así como de su influencia recíproca, los instrumentos que analizamos, según puede observarse en la tabla 2, la reglamentan. Aunque el articulado de los mismos, en el ámbito de la privacidad, es sumamente amplio, hemos optado por referenciar algunas de las cuestiones que hemos considerado más relevantes. Se ha optado por realizar la comparativa de los sellos que operan en el contexto del territorio español. En línea con cuanto planteamos, nuestro estudio empírico también estará delimitado a este elenco de sellos. Dentro de estos últimos, debemos ser conscientes que tendrán cabida logotipos que tienen su sede en otros países como los Estados Unidos, pero que operan en España.

Tabla 2 - Comparativa relativa a la ordenación de la privacidad

\begin{tabular}{|c|c|c|c|c|c|c|c|c|c|}
\hline & లే & 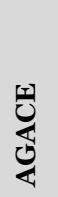 & $\begin{array}{l}\frac{0}{0} \\
\frac{\pi}{3} \\
\frac{\pi}{2} \\
0\end{array}$ & $\stackrel{\dot{0}}{0}$ & 巳્ّ & 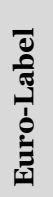 & 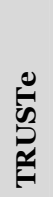 & 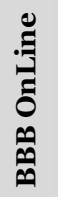 & $\begin{array}{l}\tilde{2} \\
2 \\
0 \\
0 \\
0 \\
3\end{array}$ \\
\hline $\begin{array}{l}\text { Prohibición de utilizar datos para } \\
\text { finalidades distintas a las consentidas }\end{array}$ & $\sqrt{ }$ & & $\sqrt{ }$ & & $\sqrt{ }$ & $\sqrt{ }$ & $\sqrt{ }$ & $\sqrt{ }$ & $\sqrt{ }$ \\
\hline $\begin{array}{l}\text { Cancelación de los datos ante falta de } \\
\text { necesidad, pertinencia y solicitud del titular }\end{array}$ & $\sqrt{ }$ & $\sqrt{ }$ & & & & $\sqrt{ }$ & $\sqrt{ }$ & $\sqrt{ }$ & $\sqrt{ }$ \\
\hline $\begin{array}{l}\text { Adopción de medidas técnicas y } \\
\text { organizativas que garanticen la seguridad }\end{array}$ & $\sqrt{ }$ & $\sqrt{ }$ & & $\sqrt{ }$ & $\sqrt{ }$ & & $\sqrt{ }$ & $\sqrt{ }$ & $\sqrt{ }$ \\
\hline $\begin{array}{l}\text { Comunicación de la existencia del fichero, } \\
\text { finalidad y destinatarios de la información }\end{array}$ & $\sqrt{ }$ & $\sqrt{ }$ & & & $\sqrt{ }$ & & $\sqrt{ }$ & $\sqrt{ }$ & $\sqrt{ }$ \\
\hline $\begin{array}{l}\text { Diferenciación entre campos obligatorios y } \\
\text { optativos de los formularios }\end{array}$ & $\sqrt{ }$ & $\sqrt{ }$ & $\sqrt{ }$ & & $\sqrt{ }$ & & $\sqrt{ }$ & $\sqrt{ }$ & $\sqrt{ }$ \\
\hline $\begin{array}{l}\text { Posibilidad de ejercitar los derechos de } \\
\text { acceso, rectificación, y cancelación }\end{array}$ & $\sqrt{ }$ & $\sqrt{ }$ & $\sqrt{ }$ & $\sqrt{ }$ & $\sqrt{ }$ & $\sqrt{ }$ & $\sqrt{ }$ & $\sqrt{ }$ & $\sqrt{ }$ \\
\hline $\begin{array}{l}\text { Identificación del responsable del } \\
\text { tratamiento de los datos y datos de contacto }\end{array}$ & $\sqrt{ }$ & $\sqrt{ }$ & & & $\sqrt{ }$ & & $\sqrt{ }$ & $\sqrt{ }$ & $\sqrt{ }$ \\
\hline $\begin{array}{l}\text { Información sobre la procedencia de los } \\
\text { datos obtenidos }\end{array}$ & $\sqrt{ }$ & & & & $\sqrt{ }$ & & $\sqrt{ }$ & $\sqrt{ }$ & $\sqrt{ }$ \\
\hline $\begin{array}{l}\text { Autorización previa del titular en el caso de } \\
\text { cesión de datos }\end{array}$ & $\sqrt{ }$ & $\sqrt{ }$ & $\sqrt{ }$ & & $\sqrt{ }$ & $\sqrt{ }$ & $\sqrt{ }$ & $\sqrt{ }$ & $\sqrt{ }$ \\
\hline $\begin{array}{l}\text { Autorización previa del titular en el caso de } \\
\text { uso de los datos con fines comerciales }\end{array}$ & $\sqrt{ }$ & $\sqrt{ }$ & & & & & $\sqrt{ }$ & $\sqrt{ }$ & $\sqrt{ }$ \\
\hline $\begin{array}{l}\text { Consentimiento previo del afectado para } \\
\text { tratamiento datos personales }\end{array}$ & $\sqrt{ }$ & $\sqrt{ }$ & & & $\sqrt{ }$ & & $\sqrt{ }$ & $\sqrt{ }$ & $\sqrt{ }$ \\
\hline
\end{tabular}




\begin{tabular}{lllllllllll}
\hline $\begin{array}{l}\text { Información previa sobre la existencia y } \\
\text { finalidad de cookies o similares }\end{array}$ & $\sqrt{ }$ & $\sqrt{ }$ & $\sqrt{ }$ & & $\sqrt{ }$ & & $\sqrt{ }$ & $\sqrt{ }$ & $\sqrt{ }$ \\
\hline $\begin{array}{l}\text { Mecanismos de seguridad que aseguren la } \\
\text { confidencialidad e integridad }\end{array}$ & $\sqrt{ }$ & & $\sqrt{ }$ & $\sqrt{ }$ & $\sqrt{ }$ & & & $\sqrt{ }$ & $\sqrt{ }$ & $\sqrt{ }$ \\
\hline $\begin{array}{l}\text { Medidas de protección específicas para los } \\
\text { menores de edad }\end{array}$ & $\sqrt{ }$ & $\sqrt{ }$ & $\sqrt{ }$ & & $\sqrt{ }$ & $\sqrt{ }$ & $\sqrt{ }$ & $\sqrt{ }$ & $\sqrt{ }$ \\
\hline
\end{tabular}

Todos los sellos de confianza, con buen criterio, establecen la necesidad de observar la normativa imperante en el ámbito de la privacidad. En línea con tal estipulación, es preceptivo que el personal esté suficientemente formado al respecto. Aunque podría reputarse una cuestión obvia, no ha de desmerecerse su relevancia, ya que existe un alto porcentaje de sitios Web que incumplen la legislación. De esta manera, cuando un potencial consumidor o usuario visualice, en una página, el icono acreditativo de la adhesión al documento de buenas prácticas, podrá tener garantías de que el empresario en cuestión respetará su privacidad. Los más garantistas resultan ser los tres estadounidenses (Truste, BBB Online y Web Trust) que operan en España. Los españoles son relativamente heterogéneos entre sí, siendo el más garantista Confianza Online. De manera acertada, los logotipos insisten, por un lado, en la obligación de proteger a los menores y los incapacitados y, por otro, en la necesidad de fomentar políticas de educación de los usuarios en el ámbito de la privacidad. La práctica totalidad de sellos prohíben la remisión de comunicaciones comerciales no solicitadas -spam-. De la misma manera, se impide el recurso a técnicas ideadas para tal fin, cual la recopilación de direcciones electrónicas presentes en determinados canales -address harvesting-, como la creación aleatoria de direcciones -dictionary attacks-. Finalmente, no se permite, por sus efectos negativos sobre la privacidad, la publicidad potencialmente invasiva en foros, chats y otros canales específicos.

\section{Definición de la muestra y metodología utilizada}

Teniendo en consideración la relevancia que, en el plano teórico, tienen los distintivos de calidad, relativos a la privacidad, exhibidos en los sitios Web de las empresas que operan en Internet, nos planteamos valorar las consecuencias de la adhesión. Hemos realizado un estudio empírico en el que medimos los efectos que la adhesión de este tipo de logotipos gráficos comporta para los principales destinatarios de los mismos, sus consumidores o usuarios.

El instrumento de medida utilizado ha sido una encuesta semiestructurada con preguntas cerradas y preguntas abiertas. Hemos consultado solamente a usuarios de España que hayan realizado compras a través de Internet en los últimos 12 meses, a los cuales se les ha garantizado el anonimato. Los individuos fueron segmentados por edad, nivel socio-cultural y el uso que le dan a Internet. El desarrollo del cuestionario contó con la presencia de representantes de todos los stakeholders, y se recurrió a técnicas de focus group y de votación múltiple. El constructo a medir en esta ocasión fue la privacidad. La medición se ha llevado a cabo durante 8 meses, de los cuales los 2 primeros meses se han utilizado para ajustar y validar la encuesta por medio de una muestra piloto de 50 usuarios y durante los 6 meses restantes se midió a una muestra representativa usuarios. El cuestionario inicial constaba de 75 preguntas. En el 
cuestionario final solamente quedaron 42 preguntas, la fase de fiabilidad y de validación del constructo superó todos los tests necesarios (Alpha de Cronbach, Perason, etc.). El tiempo necesario para contestar al cuestionario se estimó en 12 minutos. Se han recogido 870 respuestas válidas, la muestra es representativa por segmentos y el estudio tiene un error muestral por debajo del $3 \%$, en cada segmento, para una fiabilidad estadística del 95.5\%.

En el estudio se incluyeron variables de caracterización de los usuarios y las variables que definen los aspectos descriptivos, privacidad, lealtad/fidelidad, confianza, de los sellos más significativos que operan en el escenario español. Estos últimos no son únicamente de origen español, dado que, como vimos, ciertos prestadores de servicios radicados en España han asumido el compromiso de exhibir logotipos de privacidad que tienen su sede en otros países como los Estados Unidos.

\section{Análisis de resultados}

A continuación, presentaremos los principales resultados obtenidos a partir de la muestra seleccionada. Se han escogido usuarios de España que hayan realizado compras a través de Internet en los últimos 12 meses segmentados por edad, nivel socio-cultural y uso que le dan a Internet. Se han recogido 870 respuestas válidas, la muestra es representativa por segmentos. El estudio tiene un error muestral por debajo del $3 \%$, en cada segmento, para una fiabilidad estadística del 95.5\%.

\subsection{Análisis exploratorio de la muestra}

Se ha realizado el análisis univariante de las variables de caracterización. A continuación, se presentan los resultados más importantes, comenzaremos por los resultados de las variables de caracterización de la muestra:

Gráfico 2 - Frecuencia de uso de Internet

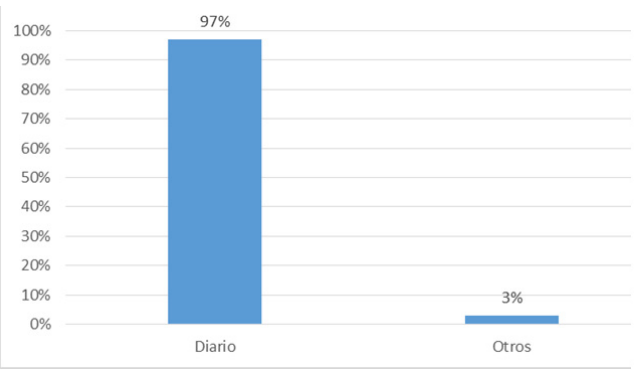

Gráfico 3 - Nivel socio-cultural

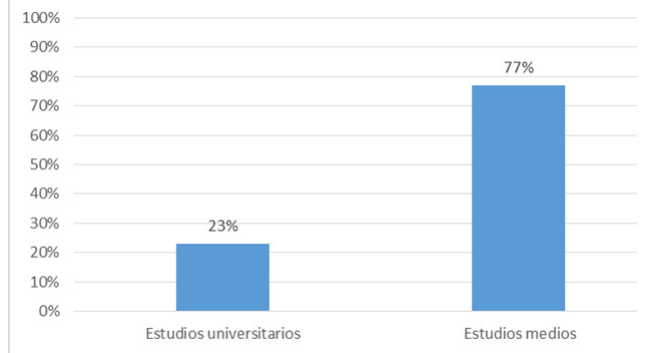


Gráfico 3 - Rango de edades

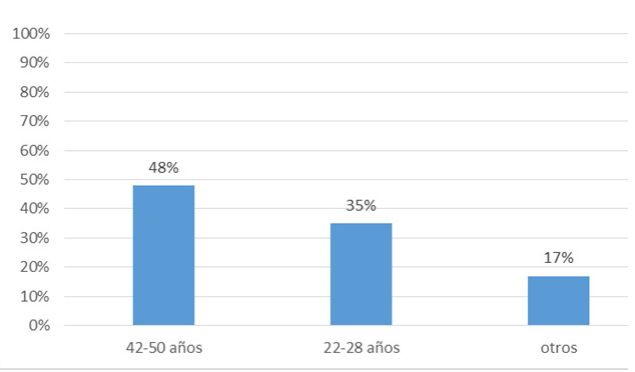

Gráfico 4 - Conocimientos de Internet

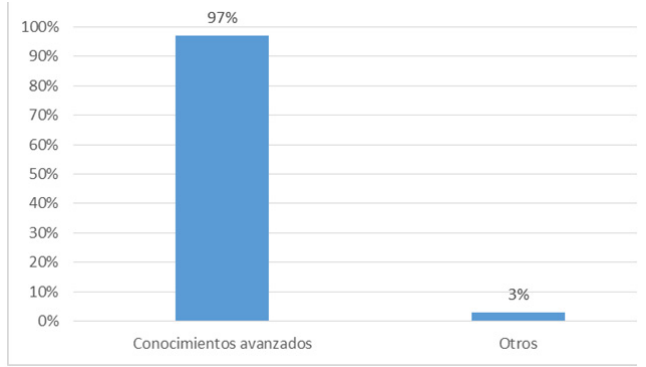

Además se han obtenido los siguientes resultados relacionados a los aspectos que definen la privacidad:

- El $82 \%$ de los encuestados considera la privacidad como el factor crítico de éxito determinante, de cara a su lealtad/fidelidad con la tienda online (recompra y recomendación). Esa cifra asciende al 96\%, si se trata de los encuestados que se encuentran por encima de los 40 años de edad (se trata del $45 \%$ de la muestra).

- El 92\% de los encuestados realiza, al menos, una operación comercial por semana en Internet.

- Asimismo, el $64 \%$ de los entrevistados considera, con carácter general, importante el hecho de que el empresario que opera en la Red esté adherido a un logotipo de privacidad. Tal cifra llega hasta el 87\% en el colectivo de 42-50 años que, además, tienen estudios universitarios. Ahora bien, debe destacarse que, aunque la mayoría de los usuarios que conocen los sellos de privacidad los valoran positivamente, el $88 \%$ no saben las diferencias que, en el ámbito de la privacidad, existen entre los mismos y los beneficios que, en su caso, ello puede determinar a su favor.

- $\quad$ El $64 \%$ de los encuestados ha comprado en, al menos, 10 tiendas online en los últimos 12 meses.

- Las transacciones más frecuentes fueron las compras de pasajes de transporte y las operaciones bancarias.

- $\quad \mathrm{Al} 36 \%$ de los encuestados realiza compras en tiendas online extranjeras.

- $\quad \mathrm{El} 43 \%$ de los encuestados no tiene reparo en realizar las compras en idioma inglés.

Como resumen, podemos decir que se trata de un grupo de personas expertas en el ámbito de Internet, que tiene adquirido el hábito de comprar, de forma online, muy adquirido. Asimismo, la tutela de la privacidad es un elemento relevante en las actuaciones que se acometan en la Red, a lo que coadyuva la concurrencia en el sitio Web del empresario de un logotipo de confianza sobre el particular. 


\subsection{Análisis multivariante de la muestra}

Se ha realizado un análisis multivariante de los aspectos encuestados (la empresa con la cual se está operando, el sello de privacidad, la transparencia -claridad y simplicidad de la Web-, el producto y la confianza que genera la empresa-sello). Las técnicas utilizadas han sido el análisis de correlación canónica, el análisis jerárquico (tipo AMOS) y las regresiones ordinales multivariantes. El propósito de esta etapa de análisis fue poder detectar las relaciones existentes entre variables y encontrar el peso (la importancia) que cada aspecto tiene en la construcción de la privacidad de los consumidores. A continuación, se presentan los resultados más importantes del análisis multivariante. Del análisis de correlación canónica y del análisis jerárquico se deduce que existen fuertes relaciones entre diversas variables. Seguidamente, se presentan esas relaciones:

Gráfico 5 - Resultados del análisis jerárquico del modelo de privacidad

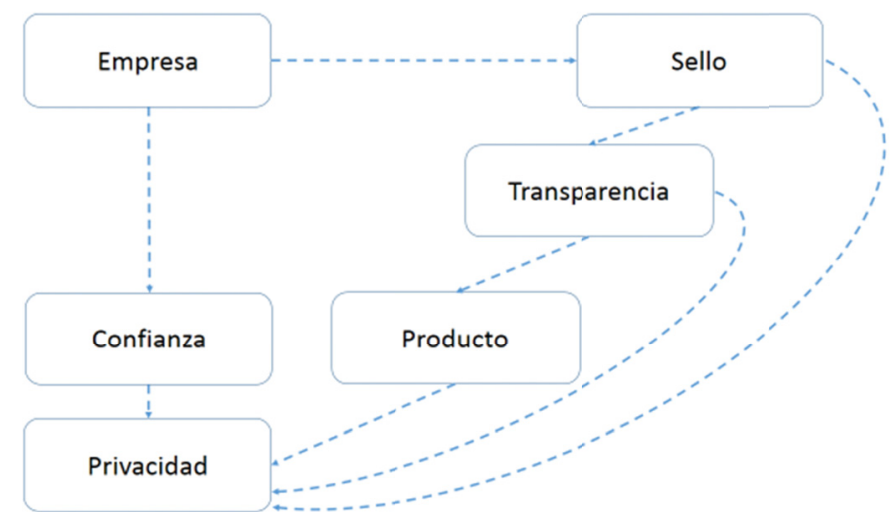

A partir de estos resultados, y para completar el estudio empírico, se han calculado los pesos, la importancia, cada uno de los aspectos analizados tiene en la creación de la confianza hacia la empresa y de la privacidad del usuario. En la tabla 3 se presentan los pesos resultantes.

Tabla 3 - Cálculo de los pesos asignados por los usuarios a cada aspecto en la privacidad de los usuarios

\begin{tabular}{lc}
\hline Aspecto & Peso \\
\hline Empresa & $26 \%$ \\
\hline Sello de privacidad & $23 \%$ \\
\hline Confianza & $21 \%$ \\
\hline Transparencia & $16 \%$ \\
\hline Producto & $14 \%$ \\
\hline Total & $100 \%$ \\
\hline
\end{tabular}


Como resumen, podemos decir que para los usuarios encuestados la privacidad depende ante todo de la empresa con la cual se está operando, seguido inmediatamente del sello de privacidad y cabe recalcar que el logotipo está por delante del producto en sí mismo y que no relacionan la confianza que les pueda inspirar la operación con el producto.

\section{Conclusiones}

En Internet impera, por diversos factores, una elevada sensación de desconfianza. Esta última se encuentra muy relacionada con las violaciones que se presentan en el ámbito de la privacidad. Frente a tal problemática se plantean, como solución verdaderamente paradigmática, los logotipos de garantía, los cuales respaldan, además del cumplimiento que, en materia de privacidad, se exige, por la legislación aplicable, un plus adicional de protección en claro beneficio del consumidor o usuario.

No todas las empresas que operan en Internet han estado dispuestas a unirse a algún sistema de confianza -en el que se incluyen los logotipos de calidad-. Ante la imposibilidad que, en la actualidad, existe de asegurar un control relativamente efectivo de las prácticas empresariales, entendemos que los iconos de confianza son un instrumento apto (valorado, en consecuencia, por el destinatario) para el consumidor o usuario, para otras empresas, e, incluso, para la propia Administración -y la sociedad, en general-, de forma tal de poder estar en condiciones de discernir a aquellos que están adheridos a los sistemas de confianza y que tutelan, de forma relevante, su privacidad. El papel de los logotipos de privacidad, como se vio en el estudio empírico, resulta determinante, y es sensiblemente valorada su presencia en cualquier sitio Web.

Respecto a los trabajos futuros que se podrían efectuar para fortalecer esta área del conocimiento, consideramos que, entre otros, cabría analizar los mecanismos en virtud de los cuales sería susceptible de alcanzar la armonización de los documentos de buenas prácticas en los que se sustentan los logotipos de calidad relativos a la privacidad. Todo ello con la finalidad de acabar con el alto grado de heterogeneidad que impera por parte de los mismos. Igualmente, entendemos que el citado estudio podría ampliarse a otros entornos y públicos. De esta manera, se podrá realizar una evaluación más amplia. Además, en este orden de cuestiones, un estudio de tipo longitudinal sería más adecuado en cuanto a la posibilidad de identificar la evolución que existe en la preocupación por la privacidad del consumidor o usuario.

\section{Referencias bibliográficas}

Aljifri, H. A., Pons, A. \& Collins, D. (2003). Global e-commerce: a framework for understanding and overcoming the trust barrier. Information Management \& Computer Secrity, 11(2), 130-138.

Belanger, F. Hiller, J. \& Smith, W.J. (2002) Trustworthiness in electronic commerce: the role of privacy, security, and site attributes. Journal of Strategic Information Systems, 11, 245-270.

Cashell, J.D. (1999). WebTrust: A seal of approval. The Internal Auditor, 56(3), 50-53. 
Caudill, E.M., \& Murphy, P.E. (2000). Consumer Online Privacy: Legal and Ethical Issues. Journal of Public Policy \& Marketing, 19 (1), 7-19.

Gefen, D. Karahanna, E. \& Straub, D.W. (2003). Trust and TAM online shopping: An integrated model. MIS Quarterly, 27(1), 51-90.

Ho, B.C. \& Oh, K.B. (2009). An empirical study of the use of e-security seals in ecommerce. Online Information Review, 33(4), 655-671.

Jawahitha, S. (2004). Consumer protection in E-commerce: Analyzing the Statutes in Malasya. Journal of American Academy of Business, 4(1), 55-63.

Kaihong, X. \& Mingxia, W. (2007). Economic Function of Trust Seal in E-Commerce: An Experiment Study Based on Chinese Subjects. Service Systems and Service Management, 9, 1-5.

Kim, D.J., Ferrin, D.L. \& Rao, H.R. (2007). A trust-based consumer decision making model in electronic commerce: the role of trust, risk, and their antecedents. Decision Support Systems, 44(2), 544-564.

Kimery, M.K. \& McCord, M. (2006). Signals of Trustworthiness in e-commerce: Consumer Understanding of Third Party Assurance Seals. Journal of Electronic Commerce in Organizations, 4(4), 52-74.

Lala, V. Arnold, V. Sutton, S. \& Guan, L. (2002). The impact of relative information quality of e-commerce assurance seals on Internet purchasing behavior. International Journal of Accounting Information Systems, 3(4), 237-254.

McKnight, D. H., Cummings, L. L. \& Chervany, N. L. (1998). Initial trust formation in new organizational relationships. Academy of Management Review, 23(3), 473490.

Mcknight, D.H. Choudhary, V. \& Kacmar, C. (2004). Shifting Factors and the Ineffectiveness of Third Party Assurance Seals: A Two-Stage Model of Initial Trust in an E-Vendor. Electronic Markets, 14(3), 252-266.

Miyazaki, A.D. \& Krishnamurthy, S. (2002). Internet seals of approval: Effects on online privacy policies and consumer perceptions. The Journal of Consumer Affairs, 36(1), 28-49.

Noteberg, A. Christiaanse, E. \& Wallage, P. (2003). Consumer trust in electronic channels: the impact of electronic commerce assurance on consumers' purchasing likelihood and risk perceptions. e-Service Journal, 2(2), 46-67.

Odom, M.D. Kumar, A. \& Saunders, L. (2002). Web assurance seals: how and why they influence consumers' decisions. Journal of Information Systems, 16(2), 231-250.

Raab, C. (1997). Co-Producing Data Protection. International Review of Law Computers \& Technology, 11(1), 11-24.

Sirkka L. Jarvenpaa and Ann Majchrzak. (2010). Vigilant Interaction in Knowledge Collaboration: Challenges of Online User Participation Under Ambivalence. Information Systems Research 21(4), 773-784. 
Teece, D.J. (2010). Business Models, Business Strategy and Innovation. Long Range Planning, 43, 172-194.

Trites, G. Boritz, J.E., \& Pugsley, D. (2006). E-Business: A Canadian Perspective for a Networked World. Toronto: Prentice Hall.

Zhan, J. \& Rajamani, V. (2008). The Economics of Privacy-Privacy: People, Policy and Technology. International Journal of Security and its Applications, 2(3), 101-108. 